depression was $20 \%$ and $25 \%$ among T2T achievers; and $31 \%$ and $38 \%$ among T2T failures, respectively $\left(P_{A}<0.05, P_{D}<0.05\right)$.

According to DAS28, the prevalence of anxiety was $19 \%, 20 \%, 27 \%$ and $41 \%$ in Remission (Rem), low disease activity (LDA), moderate disease activity (MDA) and high disease activity (HDA) subgroups respectively, the prevalence of depression was $24 \%, 26 \%, 33 \%$ and $49 \%$ respectively. The correlation coefficients of anxiety (A) and depression (D) with DAS28 were $r_{A}=0.9318$ and $r_{D}=0.9334$, respectively. It suggested that with the increase of disease activity, the proportion of RA patients with anxiety and depression increased significantly.

Combined with physical function evaluation results, the overall prevalence of anxiety (23.65\%) and depression (33.20\%) in the normal HAQ group was significantly lower than that in the abnormal HAQ group (A: $36.37 \%$, $\left.D: 43.19 \%, x_{A}^{2}=4.52, x_{D}^{2}=6.21, p_{A}<0.05, p_{D}<0.05\right) .30 .50 \%$ of the patients with $\mathrm{HAQ}=0$ and $\mathrm{DAS} 28<=2.6$ were still depressed. The analysis showed that these subgroup patients comorbid with other rheumatic diseases (SS 37\%, SLE 15\%) or suffer adverse events (abnormal WBC count: 19\%, abnormal liver function: 15\%) during treatment.

Conclusion: Higher prevalence of anxiety and depression were associated with higher levels of disease activity and worse physical function. Rheumatic comorbidity and adverse events (ADEs) were potentially associated with depression in the Rem subgroup patients with normal physical function. SSDM is an effective mobile interface to monitor and study entanglement of disease activity, physical function and mental health in RA patients, which build a foundation for proactive interventions in future. Disclosure of Interests: None declared

DOI: 10.1136/annrheumdis-2019-eular.6787

\section{AB0358 WRIST BONE MINERAL DENSITY AND WRIST SYNOVITIS IN RHEUMATOID ARTHRITIS}

Kaouther Ben Abdelghani ${ }^{1}$, Makhlouf yasmine ${ }^{1}$, Saoussen Miladi ${ }^{1}$, Alia Fazaa ${ }^{1}$, Leila Souebni ${ }^{1}$, Selma Chekili ${ }^{1}$, Kmar Ouenniche ${ }^{1}$, Salma Kassab ${ }^{1}$,

Sonia Kechaou², Zakraoui Leith ${ }^{1}$, Ahmed Laatar ${ }^{1}$. 'Mongi Slim Hospital, Tunis, Tunisia; ${ }^{2}$ Institut Pasteur, Tunis, Tunisia

Background: Bone involvement in rheumatoid arthritis is characterized by focal articular bone loss around inflamed small joints of the hands.[1] Therefore, precise quantification of hand bone loss may predict the severity and the progression of the disease.

Objectives: The aim of this study is to find a correlation between wrist bone mineral density (BMD) and sonographic wrist synovitis in patients with rheumatoid arthritis (RA).

Methods: RA patients were examined by bone mineral density (BMD) measurement of the wrist using dual-energy X-ray absorptiometry (DXA) according to standard protocols for positionning and analysis (area measured at a level of $33 \%$ ). Patients were diagnosed as having osteoporosis when the minimum $\mathrm{T}$-score measured was less than $-2.5 \mathrm{SD}$, osteopenia if T-score between $-2,5$ and -1 SD [2]. Ultrasound of the wrist was done by an operator with experience in musculoskeletal ultrasound with an esaote My lab60 machine. The wrist joint (inferior radio-ulnar joint, mediocarpal joint and ulnar recessus) was assessed on dorsal side in Gray and Power Doppler (PDUS) scales. Semi-quantitative score was used to sum the synovitis score.[3]

Statistical analysis comparing synovitis and BMD was performed using Kruskal Wallis Test, a non-parametric test (Mann-Whitney U-test) and chi-square, as appropriate.

Results: The study included 24 RA patients with female predominance (sex ratio $=0,3$ ). The mean age was 59.3 [47-71] years-old. The duration of the disease was 10.2 years $[0,83-23]$ and the body mass index was $27.3(18.97-36.98) \mathrm{kg} / \mathrm{m} 2$. RA was erosive in $75 \%$ of cases. Sixty-seven percent of patients received calcium supplementation. All women experienced already menopause. Most of them (75\%) were on corticosteroids at the average dose of $5 \mathrm{mg}$ [5-15], two of whom were not supplemented. Only one quarter of patients had physical activity. BMD showed that $45 \%$ of patients suffered from osteoporosis and $25 \%$ are osteopenic with a mean T-score of $-1.92[-4.3 ; 1.4]$ and mean bone mass of 0.475 $\mathrm{g} / \mathrm{cm}^{2}[0.239,0.712]$. Forty-eight wrists were assessed by ultrasound. Mediocarpal joint was the most affected in Gray scale and Doppler. Fifty-eight percent of patients had moderate to severe synovitis when combined scoring was used. Twenty nine percent of patients $(29,1 \%)$ had grade 3 at Gray Scale and $25 \%$ had Doppler scale grade 3.The wrist BMD (Tscore) in RA with high disease activity was lower than those with moderate or low disease activity $(-2.21$ vs -2.05 and $-2,21$ vs 0.6 respectively; $p=0,431)$. There was no correlation between BMD of the wrist and the disease duration as well as the activity of the disease DAS28 VS (respectively $p=0.4, p=0.58$ ). The percentages of wrist synovitis in osteoporosis group were significantly higher than in osteopenia group $(81.8 \%$ vs. $66 \%, p=0,03)$.Moroever, Gray scale grade 3 synovitis in osteoportic patients was higher than osteopenic patients $(62,5 \%$ vs $25 \%$ respectively, $\mathrm{p}=0,041)$. Results also showed a correlation between BMD and doppler mode grade 3 ( $83 \%$ vs 16,7 respectively, $p=0,014$ ).

Conclusion: This study shows a highly significant correlation between the BMD of the wrist and synovitis. Local inflammation of the wrist is an important factor of local bone loss that should be acted upon in order to avoid fractures, especially Colles' fracture.

\section{REFERENCES}

[1] Hansen M,et al. Bone loss in rheumatoid arthritis.. Scand J Rheumatol. 1996;25:367-376

[2] World Health Organization (2004) WHO scientific group on the assessment of osteoporosis at primary health care level.p. 5-7.

[3] D'Agostino M L, et al.Scoring ultrasound synovitis in rheumatoid arthritis : a EULAR-OMERACT ultrasound taskforce-Part 1: definition and development of a standardised, consensus-based scoring system.RMD Open; 2017;000428.

Disclosure of Interests: None declared

DOI: 10.1136/annrheumdis-2019-eular.6986

\begin{tabular}{|l|l}
\hline AB0359 & TREATING STRATEGY FOR ELDERLY RHEUMATOID \\
ARTHRITIS PATIENT, ESPECIALLY WHOSE AGE IS \\
MORE THAN 75
\end{tabular}

Ichiro Yoshii', Tatsumi Chijiwa' ${ }^{2}$, Naoya Sawada ${ }^{3} .{ }^{1}$ Yoshii Hospital, Rheumatology and Musculoskeletal Medicines, Shimanto City, Japan; ${ }^{2}$ Kochi Memorial Hospital, Rheumatology, Kochi, Japan; ${ }^{3}$ Doha Onsen Hospital, Rheumatology, Matsuyama, Japan

Background: A population of elderly rheumatoid arthritis (ERA) is increasing, probably due to treatment developed and simply elderly population increase. In aging, functional activity in daily life, immunity, especially $T$ cell function, and neural response decline and deteriorations become manifested. Treatment must not be same as that of young patient. Objectives: To evaluate our treatment method and strategy for ERA. Methods: From August 2010 to July 2015, 576 patient who have been treated in the institute continuously for more than 3 years were referred. In these, patients were classified in according with age at baseline (BL); younger than $65(\mathrm{G}-\mathrm{Y})$, from 65 to $74(\mathrm{G}-\mathrm{O})$, and no less than $75(\mathrm{G}-$ OO). Mean 28-joints disease activity score (DAS28), Health Assessment Questionnaire Disability Index (HAQ), Pain Score with visual analog scale (PS-VAS), drug administration history and dosage, were recorded.

For ERA, we have adopted a treating strategy called "Touch Down Strategy", what configures three tactics; 1) From BL, methotrexate (MTX) $6 \mathrm{mg} /$ week or tacrolimus (TAC) $1.5 \mathrm{mg} /$ day administer. 2) Increase or maintain drug dosage until clinical remission is attained or start bDMARDs when remission is not attained in 3 months, and in case, glucocorticoid (GCS) administered with every other month interval. 3)When clinical remission is attained, GCS tapering started immediately and csDMARDs tapering considered. Tapering of bDMARDs is the last order.

ERA patients were treated under these tactics. Monitored DAS28, HAQ score and PS-VAS were calculated for each group and compared with ANOVA with Bonferroni correction.

Results: $\mathrm{HAQ}$ at baseline demonstrated significantly higher in G-OO than the other groups. Prevalence of DAS28 remission were $76.4 \%, 89.6 \%$, and $87.2 \%$, while mean length from BL to DAS28 remission was $2.9,2.5$ and 4.0 months for G-Y, G-O, and G-OO, respectively. bDMARDs administration ratio was $19.8 \%, 20.6 \%$, and $18.0 \%$, while mean MTX dosage was $8.6 \mathrm{mg}, 8.6 \mathrm{mg}$, and $7.4 \mathrm{mg} /$ week, for G-Y, G-O, and G-OO respectively. GCS administration ratio and mean dosage until DAS28 remission were $24.2 \%$ and $2.96 \mathrm{mg}, 38.0 \%$ and $2.41 \mathrm{mg}$, and $42.6 \%$ and $2.71 \mathrm{mg} /$ day, while after remission $19.3 \%$ and $5.68 \mathrm{mg}, 21.1 \%$ and $4.58 \mathrm{mg}$, and $26.4 \%$ and $2.14 \mathrm{mg} /$ day, for G-Y, G-O, and G-OO, respectively (Table). Conclusion: Our Touch Down Strategy can work effective for ERA, especially for elderly ERA whose age is over 75 years old. Just by doing take care for risk of comorbidities, ERA can be well controlled their disease activity. 
Table: Characteristics of G-Y, G-O, and G-OO

\begin{tabular}{lcccc}
\hline & G-Y & G-YO & G-OO & S.S. \\
\hline Cases & 207 & 142 & 227 & n.s. \\
female (\%) & 154 & 103 & 167 & n.s. \\
& $(74.4)$ & $(72.5)$ & $(73.6)$ & \\
age at onset & 48.1 & 61.8 & 71.4 & p $<0.01$ \\
age at baseline & 50.8 & 65.1 & 78.1 & p 0.01 \\
ACPA & 123.7 & 156.8 & 229.3 & n.s. \\
DAS28 at baseline & 2.7 & 3.2 & 3.1 & n.s. \\
Sharp/van de Heijde score at baseline & 30 & 46.6 & 65.6 & n.s. \\
HAQ at baseline & 0.292 & 0.478 & 0.84 & p<0.01 \\
PS-VAS at baselline & 37.3 & 41.5 & 43.4 & n.s. \\
DAS28<2.3 prevalence (\%) & 76.4 & 89.6 & 87.2 & n.s. \\
length untilDAS28<2.3 from baseline & 2.9 & 2.5 & 4 & n.s. \\
mean DAS28 at follor up & 1.6 & 1.4 & 1.5 & n.s. \\
mean DAS28 after DAS28 remission & 2.0 & 1.8 & 2.0 & n.s. \\
b-/ts-DMARD administration case and ratio (\%) & $41(19.8)$ & $29(20.6)$ & $41(18.0)$ & n.s. \\
mean MTX dosage & 8.6 & 8.6 & 7.4 & n.s. \\
GCS administration dosage and ratio (until & 2.96, & 2.41, & 2.71, & n.s. \\
DAS28 remission) & $24.2 \%$ & $38.0 \%$ & $42.6 \%$ & \\
GCS administration dosage and ratio (after & 5.68, & 4.58, & 2.14, & n.s. \\
DAS28 remission) & $19.3 \%$ & $21.1 \%$ & $26.4 \%$ & \\
\hline
\end{tabular}

Disclosure of Interests: None declared

DOI: 10.1136/annrheumdis-2019-eular.918

\section{AB0360 EVALUATION OF HEALTHCARE RESOURCE UTILISATION AND COSTS OF SJÖGREN'S SYNDROME: PATIENTS WITH RHEUMATOID ARTHRITIS TREATED WITH ABATACEPT OR ANTI-TNF DMARDS}

Evo Alemao ${ }^{1}$, Aarti Rao ${ }^{2}$, Joe Zhuo ${ }^{1}$, Chidananda Samal ${ }^{2}$, Robert Wong ${ }^{1}$, Paul Allison ${ }^{3} .{ }^{1}$ Bristol-Myers Squibb, Princeton, United States of America; ${ }^{2} \mathrm{Mu}$ Sigma, Bangalore, India; ${ }^{3}$ Statistical Horizons LLC, Ardmore, United States of America

Background: Sjögren's syndrome (SS) is considered an extra-articular manifestation of RA and is an autoantibody-mediated condition similar to RA. In an open-label, prospective, observational multicentre study, abatacept (ABA) was found to be effective for both RA and SS-related manifestations. ${ }^{1}$ However, there are limited data on the healthcare resource utilisation $(\mathrm{HCRU})$ and cost in patients (pts) with RA with SS managed with ABA compared with those managed with anti-TNFs.

Objectives: To evaluate the HCRU and cost for pts with SS associated with RA treated with ABA or anti-TNF DMARDs.

Methods: Pts ( $\geq 18$ years) from the Truven MarketScan ${ }^{\mathrm{TM}}$ administrative claims database with incident RA $(\geq 2$ claims for RA using International Classification of Diseases [ICD]-9 or ICD-10 codes and $\geq 1$ claim for a conventional DMARD), incident SS ( $\geq 1$ claim for SS using ICD-9 or ICD10 codes) and with a prescription for ABA or an anti-TNF on or after the first diagnosis of SS from Jan 2011 to Sep 2017 were included. Pts were divided into two mutually exclusive cohorts. Pts with prescriptions for both ABA and an anti-TNF were excluded. The index date was the date of ABA or anti-TNF prescription. All-cause HCRU (healthcare visits) and costs were captured during the 2-year enrolled period prior to the index date (baseline) and in the 2-year enrolled follow-up period or until the pt was taken off the index drug, whichever occurred earlier. The baseline and follow-up periods were divided into intervals of 6 months each. Total healthcare visits (inpatient, outpatient, emergency care, urgent care and pharmacy) and total healthcare costs associated with these visits were calculated for each interval. A fixed-effects model was used to compare the HCRU and costs for pts taking ABA vs anti-TNFs after controlling for baseline Charlson Comorbidity Index and other comorbidities of interest.

Table 1

Table 1. Baseline characteristics for pts in the ABA and anti-TNF cohorts

\begin{tabular}{|c|c|c|}
\hline & ABA pts $(n=148)$ & Anti-TNF pts ( $n=1219)$ \\
\hline Age, years & $56.0(11.0)$ & $53.0(11.0)^{*}$ \\
\hline Female, $\mathrm{n}(\%)$ & $136(91.9)$ & $1065(87.4)$ \\
\hline Disease duration, days & $336.0(427.5)$ & $285.0(375.2)$ \\
\hline $\mathrm{CCl}$ score & $2.3(1.6)$ & $1.8(1.2)^{\star}$ \\
\hline \multicolumn{3}{|c|}{ Mean (SD) reported, unless stated otherwise } \\
\hline
\end{tabular}

Results: Overall, 1367 pts met inclusion criteria, of which $148(10.8 \%)$ and $1219(89.2 \%)$ were treated with $\mathrm{ABA}$ and anti-TNFs, respectively. ABA (vs
anti-TNF) pts were older and had a higher comorbidity index (Table 1). Overall HCRU decreased in both the ABA and anti-TNF groups (relative to pts not being treated with biologic DMARDs). The HCRU was lower in ABA pts vs anti-TNF pts (average visits 20.0 vs 20.8, Figure 1) in the adjusted analysis. The average adjusted total healthcare cost for ABA (vs anti-TNF) pts was lower in the four 6-month cycles evaluated (average cost $\$ 17,050$ vs $\$ 27,469$; Figure 1$)$. The overall healthcare costs were primarily driven by all-cause pharmacy cost (data not shown).

Conclusion: Pts with RA with SS managed with abatacept had lower HCRU and costs relative to pts managed with anti-TNFs. Further analysis is warranted to understand the drivers of the differential economic burden among pts with RA with SS.

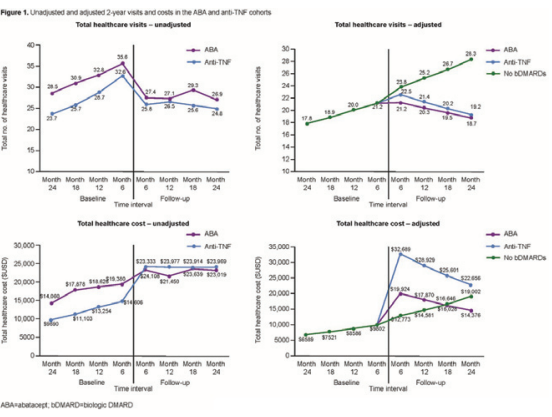

Figure 1

\section{REFERENCES:}

[1] Tsuboi H, et al. Mod Rheumatol 2016;26:891-9.

Disclosure of Interests: Evo Alemao Shareholder of: Bristol-Myers Squibb, Employee of: Bristol-Myers Squibb, Aarti Rao Consultant for: Bristol-Myers Squibb, Joe Zhuo Shareholder of: Bristol-Myers Squibb, Employee of: Bristol-Myers Squibb, Chidananda Samal Consultant for: Bristol-Myers Squibb, Robert Wong Shareholder of: Bristol-Myers Squibb, Employee of: Bristol-Myers Squibb, Paul Allison: None declared

DOI: 10.1136/annrheumdis-2019-eular.2695

\section{AB0360B ANALYSIS OF SEQUENTIAL DEVELOPMENT OF PULMONARY LESIONS IN PATIENTS WITH RHEUMATOID ARTHRITIS}

Ayae Tanaka, Yuta Takamura, Tomoyuki Miyao, Ryutaro Yamazaki, Satoko Arai, Takayoshi Owada, Reika Maezawa, Masafumi Arima, Kazuhihiro Kurasawa. Dokkyo Medical University, Rheumatology, Tochigi, Japan

Background: Pulmonary involvement is critical for the management of RA. Pulmonary involvement shows various features in pathology and imaging such as interstitial pneumonia (ILD) and airway diseases (ADs). Importantly, pulmonary abnormalities coexist with other ones. We have previously reported that there were coexpstence patterns and ADs were shared abnormalities of patients with pulmonary lesions. However, it remains unknown through what pathways various pulmonary lesions develop.

Objectives: The purpose of this study was to determine the sequential development of pulmonary abnormalities in RA.

Methods: A retrospective cohort study. Subjects were consecutive 208 RA patients who were treated with bDMARDs as the first one from Feb.2004 to Sep. 2015 in our department and received HRCT scan before and during the therapy. Based on HR-CT imaging, pulmonary abnormalities were classified into 4 categories (ILD, nodular lesions, airway disease (AD) and others) and 20 lesions such as ground-glass opacity (GGO), reticular pattern, bronchiolitis and bronchiectasis. We recorded their existence and distribution and examined their changes Cluster analysis was conducted according to new lesions at the second CT scans during the biological DMARDs, by Ward method. A checkerboard analysis with Chi-square test followed by residual analysis was conducted to examine the relation between pre-existing and newly emerging lesions. We compared the frequencies and pattern of newly emerging lesions in patients with or without pre-existing lesions.

Results: Subjects were 208 RA patients; M/F; 64/144, mean age; 59.2 years old, disease duration; 7.9 years, positive for RF in $84.1 \%$. bDMARDs used for the longest period were TNF inhibitors in $79.8 \%$ of the subjects, abatacept in $15.4 \%$ and tocilizmab in $4.8 \%$. Pulmonary 